\title{
Kondo Effect in Carbon Nanotube Quantum Dot in a Magnetic Field
}

\author{
D. KRYCHOWSKI AND S. LIPIŃSKI \\ Institute of Molecular Physics, Polish Academy of Sciences \\ M. Smoluchowskiego 17, 60-179 Poznań, Poland
}

\begin{abstract}
The out-of-equilibrium electron transport of carbon nanotube semiconducting quantum dot placed in a magnetic field is studied in the Kondo regime by means of the non-equilibrium Green functions. The equation of motion method is used. For parallel magnetic field the Kondo peak splits into four peaks, following the simultaneous splitting of the orbital and spin states. For perpendicular field orientation the triple peak structure of density of states is observed with the central peak corresponding to orbital Kondo effect and the satellites reflecting the spin and spin-orbital fluctuations.
\end{abstract}

PACS numbers: 73.22.-f, 73.23.-b, 73.63.Fg

\section{Introduction}

Carbon nanotubes (CNTs) have emerged as a viable electronic material for molecular electronic devices because they display a large variety of behavior depending on their intrinsic properties and on the characteristics of their electrical contacts [1]. These systems also form the powerful tool for the study of fundamental many-body phenomena. An example is the observed Kondo effect in semiconducting carbon nanotube quantum dots (CNTQD) $[2,3]$. The long spin lifetimes, the relatively high Kondo temperature and the fact that this effect can be seen over a very wide range of gate voltage encompassing hundreds of the Coulomb oscillations [4] make CNTQDs interesting candidates for spintronic applications.

The purpose of the present work is to discuss magnetic field dependence of the Kondo conductance of CNTQD. Perpendicular field couples only to spin and parallel field influences both spin and orbital magnetic moments. For vanishing magnetic field and orbitally degenerate states the Kondo effect appears simultaneously in spin and orbital sectors resulting in SU(4) Fermi liquid ground state with totally entangled spin and orbital degrees of freedom [5]. Magnetic field breaks the spin-orbital symmetry and in accordance to the experiment $[2,3]$ our calculations show the occurrence of the multi-peak structure of the differential conductance reflecting the spin, orbital and spin-orbital fluctuations.

\section{Model}

The low energy band structure of semiconducting carbon nanotubes is orbitally doubly degenerate at zero magnetic field. This degeneracy has been interpreted in a semiclassical fashion as the degeneracy between clockwise and counterclockwise propagating electrons along the nanotube circumference [1]. In the present considerations we restrict to the single shell and the dot is modeled by double orbital Anderson Hamiltonian with additional interorbital interaction

$$
\begin{aligned}
\mathcal{H} & =\sum_{k \alpha m \sigma} \epsilon_{k \alpha m \sigma} c_{k \alpha m \sigma}^{+} c_{k \alpha m \sigma} \\
& +\sum_{k \alpha m \sigma} t_{\alpha}\left(c_{k \alpha m \sigma}^{+} d_{m \sigma}+\text { c.c. }\right) \\
& +\sum_{k \alpha m \sigma}^{k \alpha} \epsilon_{m \sigma} d_{m \sigma}^{+} d_{m \sigma} \\
& +\sum_{m} U n_{m+} n_{m-}+\sum_{\sigma \sigma^{\prime}} U_{12} n_{1 \sigma} n_{-1 \sigma^{\prime}},
\end{aligned}
$$

where $m= \pm 1$ numbers the orbitals, the leads channels are labeled by $(m, \alpha), \alpha=\mathrm{L}, \mathrm{R} . \quad \epsilon_{m \sigma}=\epsilon_{0}+$ $\mu_{\text {orb }} m h \cos (\Theta)+g \sigma \mu_{\mathrm{B}} h, \Theta$ specifies the orientation of magnetic field $h$ relative to the nanotube axis, $\mu_{\text {orb }}$ is the orbital moment. The first term of (1) describes electrons in the electrodes, the second describes tunneling to the leads, the third represents the dot and the last two terms account for intra $(U)$ and interorbital $\left(U_{12}\right)$ Coulomb interactions. Current flowing through CNTQD can be expressed in terms of the Green functions [6]:

$$
\begin{gathered}
I_{\alpha}=\frac{\mathrm{i} e}{2 \hbar} \int_{-\infty}^{+\infty} \frac{\mathrm{d} \omega}{2 \pi} \sum_{m \sigma} \Gamma_{\alpha m \sigma}(\omega) G_{m \sigma}^{<}(\omega) \\
+\Gamma_{\alpha m \sigma}(\omega) f_{\alpha}(\omega)\left[G_{m \sigma}^{+}(\omega)-G_{m \sigma}^{-}(\omega)\right],
\end{gathered}
$$

where $G^{<}, G^{+}$and $G^{-}$are lesser, retarder and advanced Green functions, respectively, $f_{\alpha}$ is the Fermi function of $\alpha$ lead and tunneling rate $\Gamma_{\alpha m \sigma}=2 \pi\left|t_{\alpha}\right|^{2} \varrho_{\alpha m \sigma}$, where $\varrho_{\alpha m \sigma}$ is the density of states of the leads. The total current is given by $I=\left(I_{\mathrm{L}}-I_{\mathrm{R}}\right) / 2$. The lesser Green function $G^{<}$is found using $\mathrm{Ng}$ ansatz [7], according to 
which the lesser self-energy $\Sigma^{<}$is proportional to the self-energy of the corresponding noninteracting system $\Sigma^{<}(\omega)=A \Sigma_{0}^{<}(\omega)$, and $A$ can be found by the Keldysh requirement $\Sigma^{<}-\Sigma^{>}=\Sigma^{+}-\Sigma^{-}$. The Green functions are found by the equation of motion method using the self-consistent decoupling procedure proposed by Lacroix [8].

\section{Results and discussion}

The first point of our numerical analysis is addressed to the experiment of Jarillo-Herrero et al. [2], in which the conductance of CNTQD for the almost parallel field orientation was examined $\left(\Theta \approx 21^{\circ}\right)$. The calculations were performed with the Coulomb interaction parameters $U=U_{12}=40 \mathrm{meV}$, inferred from the size of the Coulomb diamonds. The addition energy spectrum indicates that the level spacing of examined CNTQDs $\Delta \epsilon \approx 4.3 \mathrm{meV}$ [9], which corresponds to the length of CNT $L \approx 400 \mathrm{~nm}$. The estimated Kondo temperature is $T_{\mathrm{K}} \approx 7.7 \mathrm{~K}[2]$. Our discussion is based on the single-shell model (1) with the level placed in the center of the Coulomb valley $\left(\epsilon_{0}=-20 \mathrm{meV}\right)$. Such an oversimplified approach, which gives only a first crude insight is justified since $\Delta \epsilon / k_{\mathrm{B}} T_{\mathrm{K}} \approx 6.5$ is large and the higher levels do not play an important role [10]. To get the experimental value of the Kondo temperature one has to assume a value of coupling to the leads $\Gamma=3.2 \mathrm{meV}$, which is slightly higher than the observed broadening of atomic or the Coulomb lines for CNTs examined by Jarillo-Herrero et al. [2, 9]. The fact that the single-level description of the multilevel systems underestimates the Kondo temperature is well known in literature $[10,11]$. Orbital moment is estimated from the average slope between the two Coulomb peaks that correspond to the addition of the electrons to the same orbital state and reads $\mu_{\mathrm{orb}} \approx 13 \mu_{\mathrm{B}}[2]$. We focus on the regime, where the quantum dot is occupied by a single electron. Figure 1a presents the calculated gray-scale plot of conductance versus magnetic field and bias voltage for $T=0.34 \mathrm{~K}$ compared with the corresponding experimental plot (inset). The central bright spot of dimension determined by $T_{\mathrm{K}}$ is the region of spinorbital Kondo effect. For vanishing bias and magnetic field the Kondo effect appears simultaneously in spin and orbital sectors resulting in a SU(4) Fermi liquid ground state. The conductance reaches in the center a value $G=1.3 e^{2} / h$. Magnetic field breaks the degeneracy and four high intensity lines appear. A pair of inner lines observed for small bias corresponds to orbital conserving fluctuations and the outer lines reflect the orbital fluctuations and simultaneous spin and orbital fluctuations. The latter two processes are not resolved for the assumed values of $\Gamma$ and temperature. Figure $1 \mathrm{~b}$ presents the linear conductance versus gate voltage at $T=8 \mathrm{~K}$ and $0.34 \mathrm{~K} . \Delta V_{\mathrm{G}}=0$ corresponds to the center of the Coulomb valley. Our calculations reasonably well reproduce the shape of the dependence but underestimate all the values of conductance roughly by a constant value
$0.5 e^{2} / h$. The source of this discrepancy is not clear, but we suggest that a possible explanation is a neglect of higher orbital levels in our description. Apart from the earlier mentioned renormalization of the Kondo temperature they also can cause a formation of shoulders in the density of states above the Fermi edge on the scale much larger than the Kondo temperature [10] and this might lead to additional weakly bias dependent contribution to the conductance. A detailed discussion of the mentioned point will be given in the following paper [12].

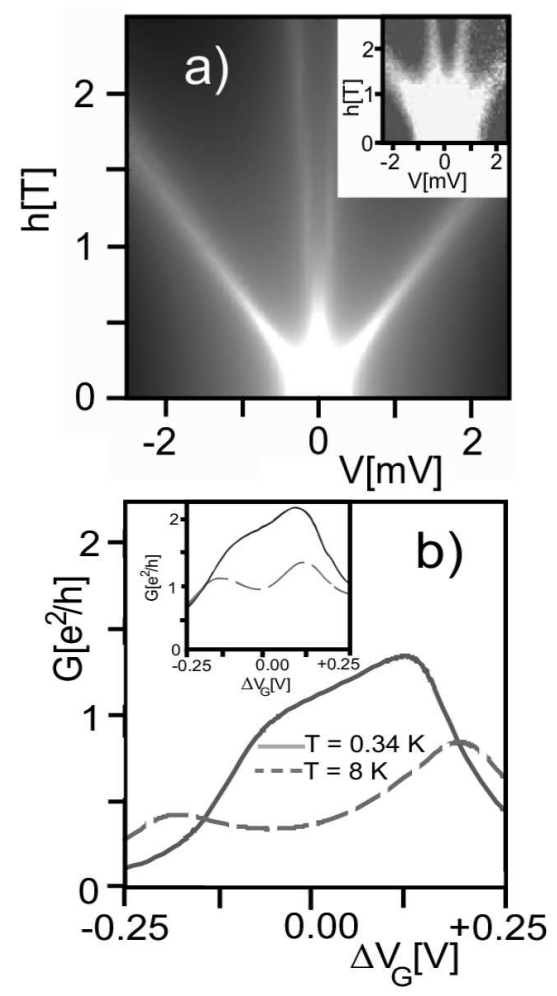

Fig. 1. (a) Calculated differential conductance $\mathrm{d} I / \mathrm{d} V$ of CNTQD versus bias voltage $V$ and magnetic field $h$ in the center of the Coulomb valley for $T=0.34 \mathrm{~K}$. The parameters used are: $U=U_{12}=40 \mathrm{meV}, \Gamma=3.2 \mathrm{meV}$, $\epsilon_{0}=-20 \mathrm{meV}$ and $\mu_{\mathrm{orb}}=13 \mu_{\mathrm{B}}$. The angle between the nanotube axis and the field $\Theta=21^{\circ}$. Gray scale: 0.1 to $1.5 e^{2} / h$. Inset shows the corresponding $(V, h)$ conductance map obtained from the data of Jarillo-Herrero et al. [2]. (b) Calculated linear conductance $G=\mathrm{d} I /\left.\mathrm{d} V\right|_{V \rightarrow 0}$ versus gate voltage at $T=0.34 \mathrm{~K}$ and $8 \mathrm{~K}$ for the CNTQD specified by parameters as in Fig. 1a. Inset shows the corresponding curves obtained from the data reported in [2].

Now let us turn to the discussion of the influence of perpendicular field, which breaks only the spin degeneracy. Our numerical analysis describes the results of Makarovski et al. [3] for $600 \mathrm{~nm}$ long nanotube quantum $\operatorname{dot}(\Delta \epsilon \approx 3 \mathrm{meV})$. The Coulomb parameters estimated again from the size of the Coulomb diamonds are taken as $U=U_{12}=15 \mathrm{meV}$ and the orbital level is placed in the center of the Coulomb valley $\epsilon_{0}=-8 \mathrm{meV} . \Gamma$ is taken as the width of orbital or the Coulomb peaks. 
$\Gamma=1.25 \mathrm{meV}$, together with the above parameters, well reproduces the experimental value of $T_{\mathrm{K}} \approx 13 \mathrm{~K}$. The calculated differential conductance for several values of magnetic field is compared with experiment in Fig. 2. A quasi $\mathrm{SU}(4)$ type behavior is still observed in the low field range, which reflects in a moderate change of conductance and a single peak structure. For higher magnetic fields the spin-orbital Kondo effect SU(4) is transformed to $\mathrm{SU}(2)$ orbital Kondo effects for each spin orientations separately. This results in the occurrence of the central peak. For bias voltage $V= \pm 2\left(\mu_{\mathrm{B}} / e\right) h$ there occur also the satellites induced by tunneling processes which mix different spin channels.

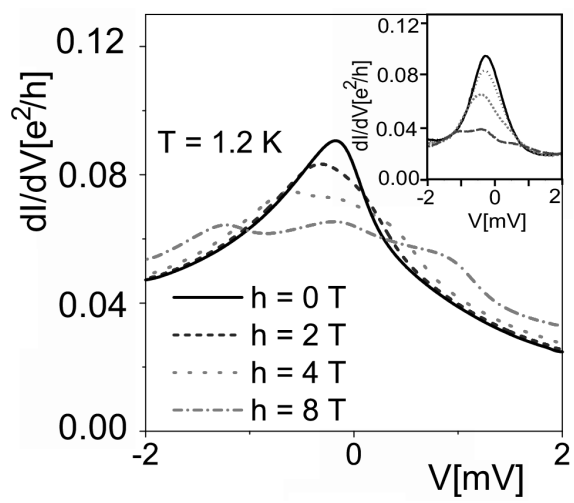

Fig. 2. Differential conductance of CNTQD in perpendicular magnetic field for $T=1.2 \mathrm{~K}$ calculated for the center of the Coulomb valley. The parameters used are: $U=U_{12}=15 \mathrm{meV}, \Gamma=1.25 \mathrm{meV}, \epsilon_{0}=-8 \mathrm{meV}$ with asymmetry of the leads $\Gamma_{\mathrm{L}} / \Gamma_{\mathrm{R}}=6$. Inset shows the corresponding curves for the same values of magnetic fields obtained from the data of Makarovski et al. [3].

Summarizing, the present paper provides a simple picture of the influence of magnetic field on the conductance of carbon nanotube QDs in the Kondo regime. Although the experiments under consideration concern multilevel dots, our calculations show that the essence of the transport properties can be inferred from the effective single shell spin-orbital Kondo physics.

\section{Acknowledgments}

This work was supported by the EU grant CARDEQ under contract IST-021285-2.

\section{References}

[1] M.S. Dresselhaus, G. Dresselhaus, Ph. Avouris, Carbon Nanotubes, Springer-Verlag, Berlin 2000.

[2] P. Jarillo-Herrero, J. Kong, H.S.J van der Zant, C. Dekker, L.P. Kouwenhoven, S. De Franceschi, $\mathrm{Na}$ ture 434, 484 (2005).

[3] A. Makarovski, A. Zhukov, J. Liu, G. Finkelstein, Phys. Rev. B 75, 241407 (2007).

[4] J. Nygard, D.H. Cobden, P.E. Lindelof, Nature 408, $342(2000)$.

[5] M. Choi, R. Lopez, R. Aquado, Phys. Rev. Lett. 95, 0672041 (2005).

[6] H. Haug, A.P. Jauho, Quantum Kinetics in Transport and Optics of Semiconductors, Springer-Verlag, Berlin 1998.

[7] T.K. Ng, Phys. Rev. Lett. 76, 487 (1996).

[8] C. Lacroix, J. Phys. F 11, 2389 (1998).

[9] P. Jarillo-Herrero, S. Sapmaz, C. Dekker, L.P. Kouvenhoven, H.S.J. van der Zant, Nature 429, 389 (2004).

[10] D. Boese, W. Hofstetter, H. Schoeller, Phys. Rev. B 66, 125315 (2002).

[11] K. Yamada, K. Yosida, K. Hanzawa, Prog. Theor. Phys. 71, 450 (1984).

[12] S. Lipiński, D. Krychowski, to be published. 